\title{
VULNERABILIDADE À PERDA DE SOLO DO PARQUE NACIONAL DA RESTINGA DE JURUBATIBA: CONTRIBUIÇÃO PARA UMA PROPOSTA DE ATRIBUIÇÃO DE PESO
}

\author{
VULNERABILITY TO SOIL LOSS OF RESTINGA DE JURUBATIBA NATIONAL \\ PARK: CONTRIBUTION TO A PROPOSAL FOR WEIGHT ASSIGNMENT \\ VULNERABILIDAD A LA PÉRDIDA DE SUELOS DEL PARQUE \\ NACIONAL DE LA RESTINGADE JURUBATIBA: CONTRIBUCIÓN \\ A UMA PROPUESTA DE ASIGNACIÓN DE PESO
}
Saulo de Oliveira Folharini - Universidade Estadual de Campinas - Campinas - São Paulo - Brasil
sfolharini@gmail.com

Regina Célia de Oliveira - Universidade Estadual de Campinas - Campinas - São Paulo - Brasil reginacoliveira@ige.unicamp.br

\begin{abstract}
André Luiz dos Santos Furtado - Embrapa Monitoramento por Satélite - Campinas - São Paulo - Brasil andre.furtado@embrapa.br
\end{abstract}

\section{Resumo}

0 estudo sistêmico procura compreender as relações existentes entre os diferentes componentes e como cada um deles atua na evolução da paisagem. Para tanto, é possível utilizar softwares de geoprocessamento que apresentam ferramentas que permitem empregar procedimentos e métodos específicos de análise de dados e informações sobre o meio ambiente. A vulnerabilidade à perda de solo tem sido investigada por meio de diferentes propostas metodológicas, entre elas, a atribuição de pesos fixos às características ambientais. Nesse caso, as características locais da área estudada não são consideradas, o que pode acarretar a obtenção de resultados imprecisos. Com o objetivo de contribuir para a discussão sobre a atribuição de pesos na análise da vulnerabilidade à perda de solo, o presente estudo propõe considerar nos cálculos a incidência espacial das classes de cada característica ambiental, tendo como área de estudo o Parque Nacional da Restinga de Jurubatiba e sua zona de amortecimento terrestre. Dessa forma, o peso torna-se variável e dependente da área, ou seja, a importância da distribuição espacial das classes de características ambientais torna-se 0 ponto fundamental para o desenvolvimento de estudos nessa temática e a metodologia pode ser aplicada em qualquer área de estudo, com pesos sendo definidos de acordo com a área de cada característica ambiental. Palavras-chave: Atribuição de peso, Jurubatiba, perda de solo, vulnerabilidade.

\section{Abstract}

The systemic study seeks to understand the relationships existing between the different components and how each one of them acts in the evolution of the landscape. To do so, it is possible to use geoprocessing software that presents tools that allow the use of procedures and specific methods of data analysis and information about the environment. Vulnerability to soil loss has been investigated through different methodological proposals, between them, the assignment of fixed weights to environmental characteristics. In this case, the local characteristics of the studied area are not considered, which can lead to inaccurate results. In order to contribute to the discussion on the attribution of weights in the analysis of vulnerability 
to soil loss, the present study proposes to consider in the calculations the spatial incidence of the classes of each environmental characteristic, having as study area the Restinga de Jurubatiba National Park and its land buffer zone. In this way, the weight becomes variable and depends on the area, it means that the importance of the spatial distribution of the classes of environmental characteristics becomes the fundamental point for the development of studies in this subject and the methodology can be applied in any area of study, with weights being defined according to the area of each environmental characteristic.

Keywords: Jurubatiba, loss of soil, vulnerability, weight assignment.

Resumen

El estudio sistémico busca comprender las relaciones entre los distintos componentes y cómo cada uno de ellos trabaja en la evolución del paisaje. Por lo tanto, es posible utilizar softwares de GIS que presentan herramientas que permiten emplear procedimientos específicos, métodos de análisis de datos e información sobre el medio ambiente. La vulnerabilidad de la pérdida de suelo se ha investigado a través de diferentes propuestas metodológicas, incluyendo la asignación de los pesos fijos a las características ambientales. En este caso, las características locales de la zona estudiada no se consideran, lo que puede dar lugar a la obtención de resultados inexactos. Con el objetivo de contribuir para la discusión sobre la atribución de pesos en el análisis de la vulnerabilidad en la pérdida de suelo, este estudio se propone considerar en los cálculos incidencia espacial de las clases de cada característica del medio ambiente, tomando como área de estudio el Parque Nacional de La Restinga de Jurubatiba y su zona de amortiguamiento terrestre. Por lo tanto, el peso se convierte en variable y depende de la zona, o sea, la importancia de la distribución espacial de las clases características ambientales se convierte en el punto fundamental para el desarrollo de los estudios sobre este tema y la metodología se puede aplicar a cualquier área de estudio, con los pesos que se determinarán de acuerdo con el área de cada característica del medio ambiente.

Palabras clave: Jurubatiba, pérdida de suelo, peso asignación,vulnerabilidad.

Introdução

A vulnerabilidade corresponde ao ponto mais fraco e com maior risco de ocorrência de um perigo. Uma denominação sintética para vulnerabilidade é a "susceptibilidade de um meio ao impacto negativo com relação a um determinado risco” (Costa et al., 2007).

Quando é relacionada ao ambiente natural, é denominada vulnerabilidade ambiental. Alguns fatores que definem a vulnerabilidade ambiental são o tipo de ocupação, eventos naturais extremos que potencializam o risco de degradação por erosão do solo, movimentos de massa, assoreamento de rios, diminuição da biodiversidade e outros processos que causam desequilíbrio no ambiente (Nascimento e Dominguez, 2009; NOAA, 1999; ONU, 2004).

De maneira geral, a paisagem mantém-se em equilíbrio dinâmico até sofrer interferência externa relacionada a um uso imposto pelo homem, que resulta em alterações nas características ambientais, modificando seu funcionamento e sua estrutura. Nesse contexto, a utilização de uma abordagem integrativa, como a teoria sistêmica, para mensurar a 
vulnerabilidade ambiental, é fundamental em estudos de diagnóstico e planejamento territorial.

Especificamente nos ambientes litorâneos, a vulnerabilidade ambiental é potencializada em decorrência de intervenções e modificações antrópicas. Na análise e na mensuração da vulnerabilidade ambiental desses ambientes, devem-se considerar as propriedades físicas, o histórico de formação e evolução do ambiente e o histórico de ocupação.

Consoante o exposto, o Parque Nacional (PARNA) da Restinga de Jurubatiba (Figura 1), localizado no litoral norte do estado do Rio de Janeiro, sofre diversas pressões antrópicas decorrentes do histórico de ocupação. O PARNA foi criado com o objetivo de proteger uma área remanescente de restinga do litoral norte fluminense e um conjunto de lagoas costeiras de relevante interesse ecológico. Dentre as diversas atividades econômicas desenvolvidas na área, nos séculos XVIII e XIX, observou-se a supressão da vegetação nativa para o cultivo de cana-deaçúcar. Posteriormente, a partir da década de 1970, com a descoberta de petróleo na Bacia de Campos, inicia-se o processo de intensificação da urbanização e da ocupação das terras da região (FEEMA, 1989).

A proposta deste estudo foi contribuir para a discussão metodológica de vulnerabilidade à perda de solo proposta por Crepani et al. (2001), considerando a incidência espacial das características ambientais; formações geológicas, compartimentos do relevo, classes de solo, precipitação, declividade, modelo digital de terreno (MDT) e uso e cobertura, na área do PARNA da Restinga de Jurubatiba, mapeadas no estudo de Folharini (2015). 


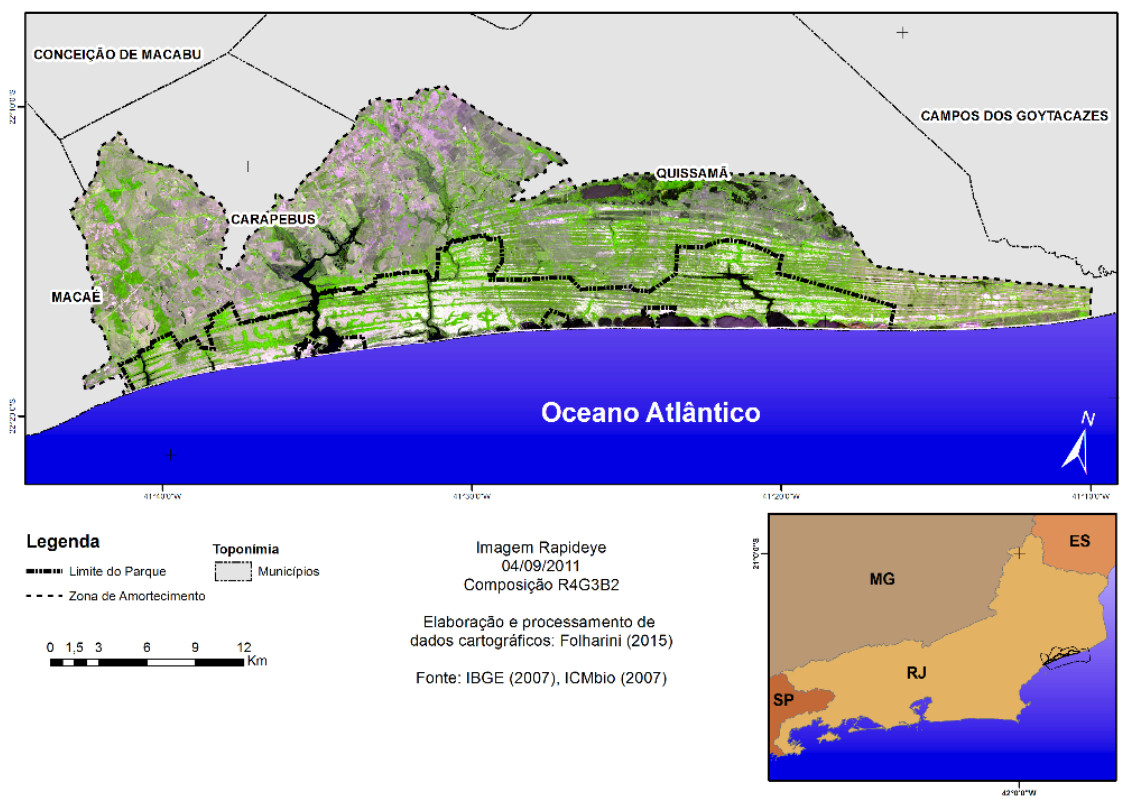

Figura 1- Localização da área de estudo

Elaboração: Folharini (2015).

O PARNA foi criado com o objetivo de proteger uma área remanescente de restinga do litoral norte fluminense e um conjunto de lagoas costeiras de relevante interesse ecológico. O mapeamento de vulnerabilidade à perda de solo auxilia na gestão da unidade de conservação, identificando o risco ao assoreamento ao qual as lagoas costeiras estão expostas, decorrente do transporte e da deposição dos sedimentos, ocasionando, dessa forma, a perda da relevância ecológica do sistema natural.

Considerando a relevância estratégica de estudos que analisam as características ambientais e de uso e cobertura da paisagem, a utilização dos sistemas de informações geográficas (SIGs), como instrumento de planejamento e ordenamento do território, subsidia a conservação do meio físico e biótico. Os SIGs tratam dados geográficos e são compostos por complexos meios técnicos, logarítmicos, idiomáticos e conceitos geográficos, geodésicos, estatísticos e cartográficos. Inserem e integram informações espaciais provenientes de dados cartográficos, 
imagens de satélite e modelos numéricos e oferecem mecanismos de combinação dessas várias informações, com a utilização de algoritmos de manipulação, análise e consulta que resultam na organização de bases diversas. Concomitantemente, unificam informações e dados territoriais, possibilitando análises integradas com enfoque sistêmico, o que os torna ferramentas com grande potencial para uso no planejamento e na gestão do território (Davis e Câmara, 2001; Moreira, 2005; Rodriguez et al., 2004; Vicente e Perez Filho, 2003).

\section{Metodologia}

A proposta metodologica de Crepani et al. (2001) analisa a vulnerabilidade da paisagem à perda de solo, tomando por base Unidades Territoriais Básicas (UTBs) e considerando duas etapas. Na primeira, as UTBs são delimitadas a partir da interpretação de padrões semelhantes, variação de cores, texturas, formas e padrões de drenagem em imagens de satélite. A seguir, é realizada uma análise integrada, com base na Teoria Ecodinâmica de Tricart (1977), atribuindo pesos de 1 a 3 às características ambientais: rocha, solo, relevo (dissecação do relevo, declividade e amplitude altimétrica), vegetação e clima. Assim, cada UTB recebe um valor de vulnerabilidade resultante da média aritmética das características analisadas.A escala varia entre muito baixa $(1,0$ a 1,4$)$, baixa $(1,5 a 1,8)$, média $(1,9$ a 2,2$)$, forte $(2,3$ a 2,6$)$ e muito forte $(2,7$ a 3,0$)$.

As UTBs relacionam-se às unidades geoambientais propostas por Rodriguez et al. (2004), que sintetizam a evolução da paisagem de uma área considerando a integração de características ambientais e de uso e ocupação das terras a partir de uma visão sistêmica. A delimitação das unidades geoambientais propostas por Rodriguez et al. (2004) e das UTBs propostas por Crepani et al. (2001) converge para um ponto comum: a importância da identificação de unidades com base em uma visão holística e sistêmica da paisagem para a gestão do território.

A visão sistêmica de evolução da paisagem, seja de forma natural, seja potencializada pela ação antrópica, resulta na transferência de matéria e energia de áreas mais elevadas para áreas mais baixas do relevo. Esse processo potencializa a vulnerabilidade da paisagem à perda de solo. A visão holística é fundamental em estudos da paisagem e para a delimitação de unidades. Segundo Silva e Santos (2011, p. 33-34): 
[...] elementos que compõem a paisagem de estudo, apesar de interligados, agrupam-se diferencialmente no espaço, com elos funcionais mais fortalecidos dentro de subconjuntos do que entre eles. Assim, determinadas porções territoriais teriam características próprias, com níveis específicos de organização, de potencialidades e de fragilidades. Nessa concepção, elaborar um zoneamento significa dividir uma área em unidades territoriais (ou zonas) específicas, em função da interpretação das características estruturais e funcionais do meio, que na tomada de decisão são destinadas a determinadas atividades, quer seja para uso humano, para conservação de um conjunto de elementos ou para preservação dos recursos naturais.

Integrar características ambientais que compõem a paisagem é o método utilizado na identificação de unidades homogêneas que têm alta similaridade e coesão, individualizando-as das demais ao seu redor. $\mathrm{Na}$ gestão territorial, as unidades são áreas que podem ser planejadas de acordo com suas potencialidades e fragilidades (Silva e Santos, 2011).

Levando-se em conta o contexto explicitado acima, propõe-se uma adaptação da metodologia de Crepani et al. (2001) para que se considere a influência da distribuição espacial das unidades. Quanto maior a área ocupada por uma unidade, maior peso ela tem e, consequentemente, mais ela influencia a vulnerabilidade. Para tanto, foi utilizada a fórmula 1, na qual PC é o peso segundo (Crepani et al., 2001), AU é a área da unidade $\left(\mathrm{km}^{2}\right)$ e AT é a área total $\left(\mathrm{km}^{2}\right)$.

$$
\text { Peso }=\frac{P C x A U}{A T}
$$

A partir dessa fórmula, foi possível definir pesos para as características de acordo com sua distribuição espacial na área de estudo. Ao considerar a área de cada unidade das características ambientais, a análise da paisagem pondera que é importante conhecer o funcionamento da área de estudo e, com isso, o resultado traduz a realidade local. $\mathrm{Na}$ Figura 2, são apresentadas as etapas do processamento. 


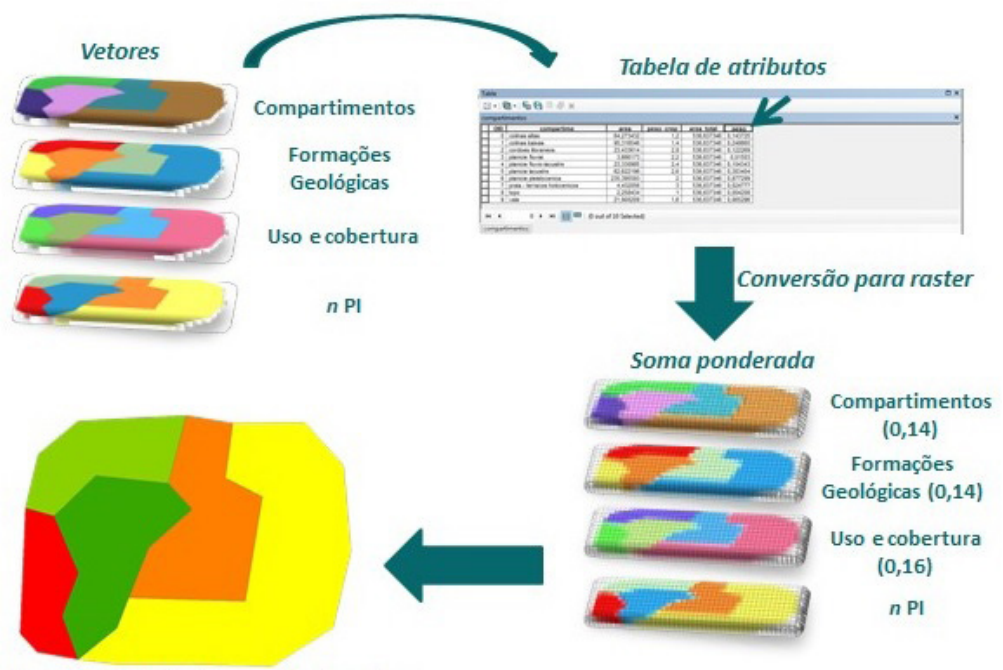

Vulnerabilidade à perda de solo

Figura 2 - Modelo simplificado das etapas de processamento. PI = Plano de Informação.

Fonte: Organizado pelos autores.

A Figura 2 apresenta as etapas de manipulação da tabela de atributos dos arquivos vetoriais, atribuindo os pesos para cada classe das características de acordo com a metodologia de Crepani et al. (2001). Em seguida, os dados vetoriais foram convertidos para raster e a soma ponderada aplicada, o que resultou no mapa de vulnerabilidade à perda de solo. A Figura 3 apresenta de forma detalhada o processamento realizado. 


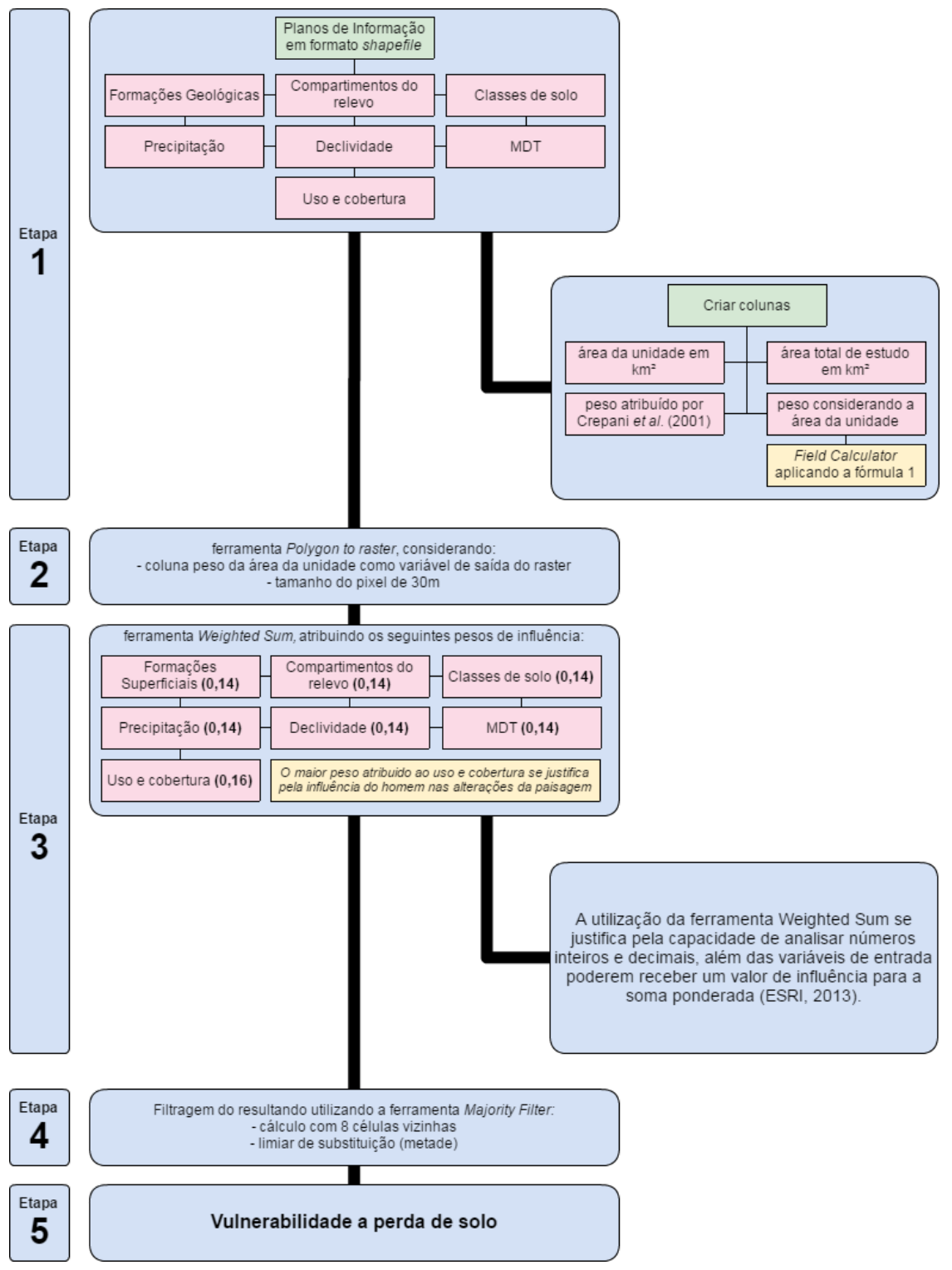

Figura 3 - Etapas da elaboração de unidades geoambientais de forma automatizada.

Fonte: Organizado pelos autores. 
Todas as etapas de processamento foram realizadas usando o software ArcGIS, versão 10.2, que agrega diferentes ferramentas de análise de dados espaciais.

\section{Resultados e discussão}

Na identificação das fragilidades e vulnerabilidades do ambiente, o principal problema observado em métodos que propõem a identificação de áreas com características comuns é a atribuição arbitrária de pesos às características de análise, como sugerido por Ross (1994) e Crepani et al. (2001). Spörl e Ross (2004, p. 48) comparam esses métodos e concluem que:

[...] pesos devem indicar a contribuição relativa de cada uma das variáveis (relevo, solo, rocha, cobertura vegetal e clima) na determinação do grau de fragilidade de uma área. No entanto, esta avaliação é geralmente arbitrária e subjetiva, pois é complicado avaliar o quanto cada uma destas variáveis contribui para se estabelecer o grau de fragilidade. Esta dificuldade em atribuir pesos a cada uma destas variáveis é que explica as divergências apresentadas entre os modelos avaliados.

A atribuição de pesos às características ambientais pode ocorrer de quatro maneiras: análise estatística, consulta aos especialistas, consulta à legislação ou consulta à literatura que já apresente um determinado peso. A análise estatística identifica situações com alta correlação das variáveis e dos fenômenos, diminuindo a subjetividade da análise, embora na escolha de um método estatístico haja uma subjetividade (Moura, 2007). Na consulta aos especialistas, existe a possibilidade de a atribuição de pesos ocorrer de forma imprecisa, resultado dos julgamentos arbitrários dos avaliadores, acarretando uma valoração numérica inconsistente dos pesos, o que compromete o conjunto da análise. Por sua vez, a determinação dos pesos com base na legislação ou na literatura está baseada em evidências empíricas, o que prejudica a aplicação em locais com características distintas da área de estudo (Coelho, 2011; Garofalo, 2013).

Considerando que a análise da paisagem fundamenta-se em uma discussão sistêmica e dinâmica, na qual a proposta de Tricart (1977) tem papel fundamental, será apresentado em seguida o resultado do processamento descrito na metodologia utilizada neste estudo.

Na etapa 1, foram definidos os pesos considerando a área de cada unidade $^{1}$ e a metodologia proposta por Crepani et al. (2001). Nas Tabelas I a 
VII, são apresentados os valores de peso atribuídos às áreas das unidades, a área total e o peso relativo considerando a área de cada unidade.

A Tabela I contempla a atribuição de pesos às formações geológicas:

\begin{tabular}{|c|c|c|c|c|}
\hline Formações geológicas & $\begin{array}{c}\text { Área da } \\
\text { unidade }\left(\mathrm{km}^{2}\right)\end{array}$ & $\begin{array}{c}\text { Peso atribuído } \\
\text { (segundo Crepani et } \\
\text { al., 2001) } \\
\end{array}$ & $\begin{array}{l}\text { Área total } \\
\left(\mathrm{km}^{2}\right)\end{array}$ & $\begin{array}{c}\text { Peso relativo } \\
\text { considerando a } \\
\text { área da unidade }\end{array}$ \\
\hline Complexo Búzios & 126,32 & 1,3 & 536,63 & 0,30 \\
\hline Complexo Região dos Lagos & 28,14 & 1,7 & 536,63 & 0,08 \\
\hline Depósitos flúvio-lagunares & 39,92 & 3 & 536,63 & 0,22 \\
\hline Depósitos lagunares & 28,37 & 3 & 536,63 & 0,15 \\
\hline Depósitos praiais marinhos & 3,45 & 3 & 536,63 & 0,01 \\
\hline $\begin{array}{l}\text { Depósitos praiais marinhos e/ou } \\
\text { lagunares }\end{array}$ & 266,28 & 3 & 536,63 & 1,48 \\
\hline $\begin{array}{l}\text { Depósitospraiais eólicos, marinhos e/ou } \\
\text { lagunares }\end{array}$ & 18,06 & 3 & 536,63 & 0,10 \\
\hline Grupo Barreiras & 8,04 & 3 & 536,63 & 0,04 \\
\hline Suíte Desengano & 17,08 & 1,7 & 536,63 & 0,05 \\
\hline
\end{tabular}

Tabela I - Pesos atribuídos às formações geológicas.

Fonte: Organizado pelos autores.

A atribuição dos pesos às formações geológicas considerou a composição litológica dominante em cada formação, conforme definido por Crepani et al. (2001) na tabela de escala da vulnerabilidade à denudação das rochas mais comuns.

A Tabela II apresenta os pesos atribuídos aos compartimentos do relevo, delimitados no estudo de Folharini et al. (2014).

\begin{tabular}{|c|c|c|c|c|}
\hline Compartimentos do relevo & $\begin{array}{l}\text { Área da unidade } \\
\qquad\left(\mathrm{km}^{2}\right)\end{array}$ & $\begin{array}{l}\text { Peso atribuído } \\
\text { (segundo Crepani } \\
\text { et al., 2001) }\end{array}$ & $\begin{array}{l}\text { Área total } \\
\left(\mathrm{km}^{2}\right)\end{array}$ & $\begin{array}{c}\text { Peso relativo } \\
\text { considerando a área } \\
\text { da unidade }\end{array}$ \\
\hline Colinas altas & 64,27 & 1,9 & 536,63 & 0,22 \\
\hline Colinas baixas & 95,31 & 2,1 & 536,63 & 0,37 \\
\hline Cordões litorâneos & 23,43 & 3 & 536,63 & 0,13 \\
\hline Planície fluvial & 3,66 & 3 & 536,63 & 0,02 \\
\hline Planície fluvio-lacustre & 23,33 & 3 & 536,63 & 0,13 \\
\hline Planície lacustre & 62,62 & 3 & 536,63 & 0,35 \\
\hline
\end{tabular}




\begin{tabular}{l|c|c|c|c}
\hline Planície pleistocênica & 235,39 & 3 & 536,63 & 1,31 \\
\hline Praia & 4,43 & 3 & 536,63 & 0,02 \\
\hline Topo & 2,25 & 1,7 & 536,63 & 0,00 \\
\hline Vale & 21,90 & 2,3 & 536,63 & 0,09 \\
\hline
\end{tabular}

Tabela II - Pesos atribuídos aos compartimentos do relevo

Fonte: Organizado pelos autores.

Para variáveis morfométricas, Crepani et al. (2001) utilizam índice de dissecação do relevo, declividade e amplitude altimétrica para calcular a vulnerabilidade da geomorfologia por meio de soma ponderada (Vulnerabilidade Geomorfologia $=$ Dissecação + Amplitude + Declividade/3). Essa metodologia define os seguintes valores de vulnerabilidade para as formas de relevo: de 1,0 a 1,6 para relevos planos a suavemente ondulados; 1,7 a 2,3 para relevos ondulados dissecados em colinas; 2,4 a 3,0 para relevos fortemente ondulados a escarpados; e 3,0 para formas de acumulação de origem fluvial, marinha ou lacustre, como planícies e terraços sujeitos à inundação, nos quais processos morfodinâmicos predominam e configuram esses meios como instáveis.

Na Tabela III são apresentados os pesos atribuídos às classes de declividade, variável morfométrica utilizada no cálculo de vulnerabilidade da geomorfologia.

\begin{tabular}{c|c|c|c|c}
\hline Declividade & Área da unidade $\left(\mathbf{k m}^{2}\right)$ & $\begin{array}{c}\text { Peso atribuído (segundo } \\
\text { Crepani et al., 2001) }\end{array}$ & $\begin{array}{c}\text { Área total } \\
\left.\mathbf{( k m}^{2}\right)\end{array}$ & $\begin{array}{c}\text { Peso relativo } \\
\text { considerando a } \\
\text { área da unidade }\end{array}$ \\
\hline$>50 \%$ & 0,65 & 3 & 536,63 & 0,00 \\
\hline $20 \mathrm{~F} 50 \%$ & 15,14 & 2,5 & 536,63 & 0,07 \\
\hline $6 \mathrm{H} 20 \%$ & 98,15 & 2 & 536,63 & 0,36 \\
\hline $2 \mathrm{H} 6 \%$ & 133,95 & 1,5 & 536,63 & 0,37 \\
\hline$<2 \%$ & 288,69 & 1 & 536,63 & 0,53 \\
\hline
\end{tabular}

Tabela III - Pesos atribuídos às classes de declividade

Fonte: Organizado pelos autores.

A última variável utilizada no cálculo de vulnerabilidade da geomorfologia é a amplitude altimétrica, informação obtida a partir do modelo digital de terreno (MDT), que é apresentada na Tabela IV. 


\begin{tabular}{c|c|c|c|c}
\hline $\begin{array}{c}\text { MDT (amplitude } \\
\text { altimétrica, em m) }\end{array}$ & $\begin{array}{c}\text { Área da } \\
\text { unidade }\left(\mathbf{k m}^{2}\right)\end{array}$ & $\begin{array}{c}\text { Peso atribuído (segundo } \\
\text { Crepani et al., 2001) }\end{array}$ & Área total $\left(\mathbf{k m}^{2}\right)$ & $\begin{array}{c}\text { Peso relativo } \\
\text { considerando a } \\
\text { área da unidade }\end{array}$ \\
\hline $132-141,5$ & 0,00 & 2,3 & 536,63 & 0,00 \\
\hline $112,5-132$ & 0,01 & 2,2 & 536,63 & 0,00 \\
\hline $113-122,5$ & 0,02 & 2,1 & 536,63 & 0,00 \\
\hline $103,5-113$ & 0,05 & 2 & 536,63 & 0,00 \\
\hline $94-103,5$ & 0,07 & 1,9 & 536,63 & 0,00 \\
\hline $84,5-94$ & 0,16 & 1,8 & 536,63 & 0,00 \\
\hline $77-84,5$ & 0,32 & 1,7 & 536,63 & 0,00 \\
\hline $67,5-77$ & 1,05 & 1,6 & 536,63 & 0,00 \\
\hline $58-67,5$ & 2,83 & 1,5 & 536,63 & 0,00 \\
\hline $48,5-58$ & 6,61 & 1,4 & 536,63 & 0,01 \\
\hline $39-48,5$ & 14,53 & 1,3 & 536,63 & 0,03 \\
\hline $29,5-39$ & 24,83 & 1,2 & 536,63 & 0,05 \\
\hline $20-29,5$ & 36,66 & 1,1 & 536,63 & 0,07 \\
\hline$<20$ & 449,43 & 1 & 536,63 & 0,83 \\
\hline
\end{tabular}

Tabela IV - Pesos atribuídos à amplitude altimétrica no modelo digital de terreno (MDT)

Fonte: Organizado pelos autores.

Os pesos referentes à vulnerabilidade das classes de solo em relação a processos erosivos foram atribuídos por Crepani et al. (2001) com base na estrutura do solo, determinada pelo padrão de arranjo e da quantidade de partículas primárias (areia, silte e argila) em agregados, que são separados por superfícies de fraqueza.

A classificação levou em conta: a estrutura dos solos, que pode ser laminar, prismática, poliédrica (blocos) e esferoidal (granular); a permeabilidade, que corresponde à capacidade do solo de transmitir fluidos como a água; a profundidade, representada pela ocorrência de materiais constituintes do solo em superfície, subsuperfície ou em maior profundidade; e a presença de camadas impermeáveis, ou seja, rochas com baixa porosidade, que dificultam a penetração da água (Santos et al., 2005).

Os valores de vulnerabilidade das classes de solo são apresentados na Tabela 4.11 da metodologia de Crepani et al. (2001) e mostrados na Tabela V: 


\begin{tabular}{l|c|c|c|c}
\hline Classes de solo & $\begin{array}{c}\text { Área da unidade } \\
\left(\mathbf{( k m}^{2}\right)\end{array}$ & $\begin{array}{c}\text { Peso atribuído } \\
\text { (segundo Crepani et } \\
\text { al., 2001) }\end{array}$ & $\begin{array}{c}\text { Área total } \\
\left(\mathbf{k m}^{2}\right)\end{array}$ & $\begin{array}{c}\text { Peso relativo } \\
\text { considerando a } \\
\text { área da unidade }\end{array}$ \\
\hline Argissolo Vermelho-Amarelo Álico & 7,70 & 2 & 536,63 & 0,02 \\
\hline Espodossolo Amarelo Álico & 97,09 & 2 & 536,63 & 0,00 \\
\hline $\begin{array}{l}\text { Espodossolo Hidromórfico } \\
\text { distrófico }\end{array}$ & 271,31 & 2 & 536,63 & 0,02 \\
\hline Gleissolo Pouco Húmico Álico & 59,09 & 3 & 536,63 & 0,27 \\
\hline Latossolo Vermelho Distrófico & 54,78 & 1 & 536,63 & 0,10 \\
\hline Organossolo Tiomórfico & 40,40 & 3 & 536,63 & 0,33 \\
\hline Areias Quartzosas Marinhas & 4,42 & 3 & 536,63 & 0,09 \\
\hline
\end{tabular}

Tabela V - Pesos atribuídos às classes de solo

Fonte: Organizado pelos autores.

Outra variável considerada foi a precipitação, uma vez que é um agente modelador do relevo. Sua escala de erosividade e seus valores de vulnerabilidade são apresentados Tabela 25 de Crepani et al. (2001) e exibidos na Tabela VI.

\begin{tabular}{l|c|c|c|c}
\hline Precipitação & $\begin{array}{c}\text { Área da } \\
\text { unidade }\left(\mathbf{k m}^{2}\right)\end{array}$ & $\begin{array}{c}\text { Peso atribuído (segundo } \\
\text { Crepani et al., 2001) }\end{array}$ & Área total $\left(\mathbf{k m}^{2}\right)$ & $\begin{array}{c}\text { Peso relativo } \\
\text { considerando a } \\
\text { área da unidade }\end{array}$ \\
\hline $75 \mathrm{~F} 100 \mathrm{~mm}$ & 372,69 & 1,2 & 536,63 & 0,83 \\
\hline $50 \mathrm{~F} 75 \mathrm{~mm}$ & 196,94 & 1,1 & 536,63 & 0,40 \\
\hline
\end{tabular}

Tabela VI - Pesos atribuídos à precipitação

Fonte: Organizado pelos autores.

Já para o uso e a cobertura da terra, Crepani et al. (2001) consideram a importância da densidade da cobertura vegetal na determinação da vulnerabilidade, uma vez que os processos erosivos são potencializados nas áreas com reduzida cobertura vegetal. Na escala proposta, Crepani et al. (2001) determinam cinco classes: estável $(1,0)$, moderadamente estável $(1,5)$, medianamente estável $(2,0)$, moderadamente vulnerável $(2,5)$ e vulnerável $(3,0)$. Alta densidade de cobertura vegetal apresenta valores próximos a 1,0; densidade intermediária, valores próximos a 2,0; e baixa densidade de cobertura vegetal, valores próximos a 3,0. Em relação às coberturas artificiais, os autores determinam os seguintes pesos: pastagens $-2,8$; culturas perenes $-2,9$; e culturas anuais $-3,0$. Na Tabela VII, são apresentados os pesos atribuídos às classes de uso e cobertura da terra. 


\begin{tabular}{l|c|c|c|c}
\hline Uso e cobertura & $\begin{array}{c}\text { Área da } \\
\text { unidade }\left(\mathbf{k m}^{2}\right)\end{array}$ & $\begin{array}{c}\text { Peso atribuído (segundo } \\
\text { Crepani et al., 2001) }\end{array}$ & $\begin{array}{c}\text { Área total } \\
(\mathbf{k m})\end{array}$ & $\begin{array}{c}\text { Peso relativo } \\
\text { considerando a } \\
\text { área da unidade }\end{array}$ \\
\hline Área agrícola & 21,93 & 3 & 536,63 & 0,12 \\
\hline Areia & 28,49 & 3 & 536,63 & 0,15 \\
\hline Florestas de terras baixas & 11,45 & 1 & 536,63 & 0,02 \\
\hline Formações arbóreo-arbustivas & 127,52 & 2,7 & 536,63 & 0,64 \\
\hline $\begin{array}{l}\text { Formações arbustivo-herbáceas } \\
\text { (moitas menores) }\end{array}$ & 30,94 & 2,3 & 536,63 & 0,13 \\
\hline $\begin{array}{l}\text { Formações arbustivo-herbáceas } \\
\text { densas (moitas maiores) }\end{array}$ & 34,86 & 2,3 & 536,63 & 0,14 \\
\hline Pastagem & 54,74 & 2,8 & 536,63 & 0,28 \\
\hline Solo exposto (área antropizada) & 116,76 & 2,9 & 536,63 & 0,63 \\
\hline \begin{tabular}{l} 
Vegetação em área úmida \\
\hline
\end{tabular} & 93,54 & 2,3 & 536,63 & 0,40 \\
\hline
\end{tabular}

Tabela VII - Pesos atribuídos ao uso e à cobertura

Fonte: Organizado pelos autores.

As informações apresentadas nas tabelas anteriores foram configuradas na tabela de atributos de cada característica ambiental analisada, por meio de inserção das colunas: área da unidade, em $\mathrm{km}^{2}$; peso atribuído por Crepani et al. (2001); área total de estudo, em $\mathrm{km}^{2}$; e peso considerando a área da unidade, calculada utilizando a ferramenta Field Calculator, com aplicação da fórmula 1.

Na etapa 2, foi utilizada a ferramenta Polygon to raster para converter os arquivos vetoriais em imagens. A coluna peso, considerando a área da unidade, foi utilizada como input para a conversão.

As imagens geradas foram então processadas (etapa 3), por meio da ferramenta Weighted Sum, aplicando uma soma ponderada às imagens. Cada característica ambiental recebeu um peso que variou de 0 a 1. Buscando manter uma distribuição igualitária, as características ambientais receberam peso 0,14, e uso e cobertura receberam 0,16. Essa distribuição decorre de o uso e a cobertura terem capacidade de modificação da paisagem - o homem torna-se um agente externo que interfere na estabilidade da paisagem e, consequentemente, na vulnerabilidade à perda de solo. Por exemplo, se uma área sofre supressão da vegetação proporcionada pelo homem, a vulnerabilidade à perda de solo aumenta nesse local. 
Em seguida, a etapa 4 empregou a ferramenta Majority Filter, para diminuir a quantidade de pixels isolados e, assim, homogeneizar a imagem. Nesta etapa, foram considerados os parâmetros eight em number of neighbors to use e half em replacement threshold.

A etapa 5 consistiu na organização do mapa final de escala de vulnerabilidade à perda de solo, no intervalo de 0 a 1.Valores próximos a 0 identificam áreas com baixa probabilidade de ocorrência de perda de solo, e valores próximos a 1, áreas com alta probabilidade de ocorrência de perda de solo. O resultado do mapeamento de vulnerabilidade à perda de solo é mostrado na Figura 4. 


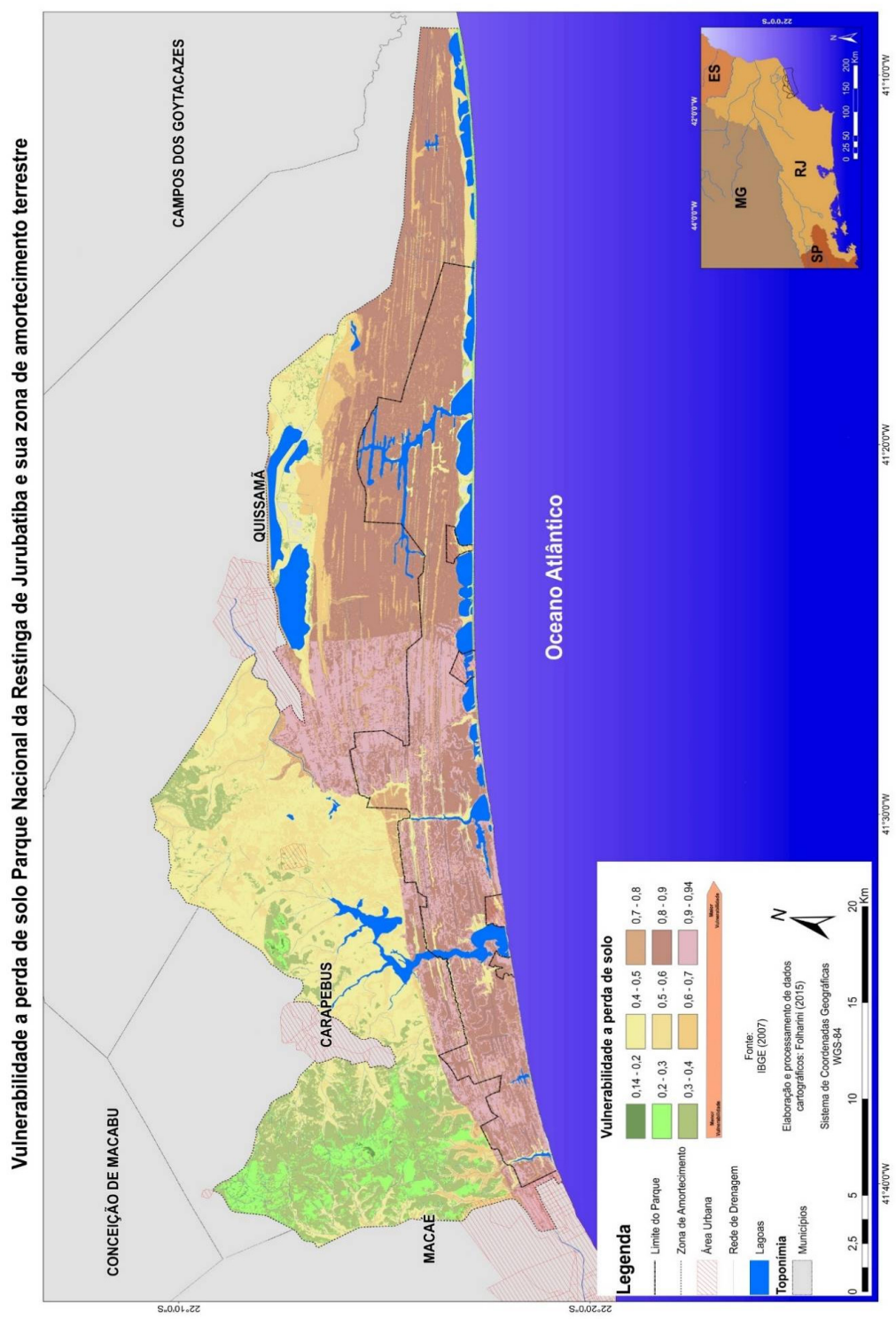

Figura 4 - Vulnerabilidade à perda de solo do Parque Nacional da Restinga de Jurubatiba e sua zona de amortecimento terrestre 
O presente estudo apresenta uma proposta metodológica para quantificar a vulnerabilidade à perda de solo, considerando a contribuição da incidência espacial de cada variável e evidenciando a situação real da área do PARNA da Restinga de Jurubatiba. Dessa forma, salienta-se que é de fundamental importância para a melhor acurácia dos resultados de uma investigação considerar as características e sua a incidência espacial em uma área de estudo.

\section{Considerações finais}

O funcionamento da paisagem é um tema multidisciplinar que abarca diferentes correntes de pensamento e técnicas de análise. Entender esse funcionamento é fundamental para propor políticas públicas de gestão territorial.

Uma dessas técnicas, a análise espacial propõe modelos que podem ser utilizados na gestão territorial. Um desses modelos, empregado em estudos e planos de gestão, é o mapeamento de vulnerabilidade à perda de solo, que identifica, em uma escala de valores, áreas mais ou menos propensas à mobilização e ao transporte do solo.

A proposta de Crepani et al. (2001) é uma metologia que define uma escala de valores para a vulnerabilidade à perda de solo e tem sido amplamente aplicada no meio científico. Nesse caso, as variáveis ambientais são definidas por pesos fixos (Spörl e Ross, 2004).

Para minimizar o efeito arbitrário na atribuição de pesos, o presente estudo considera a incidência espacial de cada unidade das características ambientais. Dessa forma, quanto maior a área da unidade, mais ela contribui para que o peso seja elevado e, quanto menor a área da unidade, menos ela contribui, definindo pesos com valores baixos.

No caso do PARNA da Restinga de Jurubatiba, as características ambientais analisadas indicam que a planície costeira, em decorrência de sua extensa área, apresenta maior vulnerabilidade à perda de solo, portanto são atribuídos pesos maiores para formações geológicas, compartimentos geomorfológicos e classes de solos compostas por matérias com pouca coesão, facilmente mobilizados pela ação da água. Por sua vez, as classes de declividade abaixo de $20 \%$ têm maior peso e também estão relacionadas às áreas de planície litorênea. 
Em relação às classes de amplitude altimétrica, Crepani et al. (2001) atribuiram um grande número de classes.Ao aplicar os pesos atribuídos pelos autores citados no PARNA da Restinga de Jurubatiba, os valores de peso considerando a área da unidade foram pequenos e concentram-se em altitudes $<20 \mathrm{~m}$.

Já a precipitação foi dividida em duas classes, 50 H75 mm e 75 F $100 \mathrm{~mm}$, com pesos considerando a área da unidade de 0,40 e 0,83, respectivamente. A influência da precipitação no processo de perda de solo é mais evidente nas regiões central e norte do PARNA, locais onde a precipitação está na classe de $75 \mathrm{H} 100 \mathrm{~mm}$.

Por fim, os valores de peso considerando a área da unidade no uso e na cobertura da terra das classes formações árboreo-arbustivas $(0,64)$, solo exposto $(0,63)$ e vegetação em área úmida $(0,40)$ demonstram grande importância tanto da atuação da vegetação quanto das áreas de solo exposto na planície costeira. Uma característica natural da vegetação de restinga é sua ocorrência esparsa, intercalada com áreas de solo exposto. Essa configuração define uma maior vulnerabilidade à perda de solo, já que o papel da vegetação de interceptar a água da chuva é reduzido.

Após processar os dados e analisar a paisagem do PARNA da Restinga de Jurubatiba, conclui-se que há duas áreas distintas de vulnerabilidade à perda de solo:1) a planície costeira, em decorrência de sua constituição por materiais inconsolidados, vegetação esparsa e atuação da precipitação; 2) áreas de colinas, em decorrência de estruturas geológica e geomorfológica mais coesas, conjugadas à cobertura vegetal e a tipos de uso, fazem com que a vulnerabilidade à perda de solo nessas áreas seja menor.

O resultado apresentado neste estudo contribui para a discussão de atribuição de peso às características ambientais, propondo uma possibilidade na atribuição e na distribuição desses valores, ao considerar a incidência espacial.

Nota

1 Os dados georreferenciados utilizados neste estudo estão disponíveis no WebGIS do projeto, no endereço <http://mapas.cnpm.embrapa.br/peld $>$. 


\section{Agradecimentos}

O primeiro autor agradece ao Conselho Nacional de Desenvolvimento Científico e Tecnológico (CNPq), pela concessão da bolsa de Desenvolvimento Tecnológico e Industrial, nível B (DTI-B), processo 380557/2015-0.

\section{Referências}

COELHO, H. M. G. Modelo para avaliação e apoio ao gerenciamento de resíduos sólidos de indústrias. 2005. 280 f. Tese (Doutorado em Saneamento, Meio Ambiente e Recursos Hídricos) - Escola de Engenharia, Universidade Federal de Minas Gerais, Belo Horizonte, 2005.

COSTA, T. C. C.; UZEDA, M. C.; FIDALGO, E. C. C.; LUMBRERAS, J. F.; ZARONI, M. J.; NAIME, U. J.; GUIMARÃES, S. P. Vulnerabilidade ambiental em subbacias hidrográficas do estado do Rio de Janeiro por meio de integração temática da perda de solo (USLE), variáveis morfométricas e o uso/cobertura da terra. In: SIMPÓSIO BRASILEIRO DE SENSORIAMENTO REMOTO, 13. (SBSR). 2007, Florianópolis. Anais... São José dos Campos: INPE, 2007. p. 2493-2500.

CREPANI, E.; MEDEIROS, J. S.; HERNANDEZ FILHO, P.; FLORENZANO, T. G.; DUARTE, V.; BARBOSA, C. C. F. Sensoriamento remoto e geoprocessamento aplicados ao zoneamento ecológico-econômico e ao ordenamento territorial. São José dos Campos: INPE, 2001. Disponível em: http://www.dsr.inpe.br/laf/sap/ artigos/CrepaneEtAl.pdf. Acesso em: 18 out. 2015.

DAVIS, C.; CAMARA, G. Arquitetura de sistemas de informação geográfica. In: CAMARA, G.; DAVIS, C.; MONTEIRO, R. F. (Eds.). Introdução à ciência da geoinformação. São José dos Campos: INPE, 2001.

FEEMA. Fundação Estadual Engenharia Meio Ambiente.Perfil ambiental: municípios de Macaé e Quissamã. Rio de Janeiro: 1989.

FOLHARINI, S. O.; OLIVEIRA, R. C.; FURTADO, A. L. S.; CONCEIÇÃO, A. F. Compartimentação geomorfológica do Parque Nacional da Restinga de Jurubatiba e sua zona de amortecimento terrestre. In XIV COLÓQUIO IBERICO DE GEOGRAFIA, 2014, Guimarães - Portugal. A jangada de pedra - geografias ibero-afro-americanas. Atas do Colóquio Ibérico de Geografia. Guimarães: Universidade do Minho, v. 1, 2014. p. 2224-2229.

FOlHARINI, S. O. Análise geoecológica do Parque Nacional da Restinga de Jurubatiba e sua zona de amortecimento terrestre utilizando geoprocessamento. 2015. Dissertação (Mestrado em Geografia) - Instituto de Geociências, Universidade Estadual de Campinas, Campinas, SP, 2015.

GAROFALO, D. F. T. Mapeamento de fragilidade ambiental por meio de análise espacial: um exemplo da alta bacia dos rios Piracicaba e Sapucaí-Mirim APA Fernão Dias-MG. 2013. Dissertação (Mestrado em Geografia) - Instituto de Geociências, Universidade Estadual de Campinas, Instituto de Geociências, Campinas, SP, 2013. 
MOREIRA, M. A. Fundamentos do sensoriamento remoto e metodologias de aplicação. 3. ed. Viçosa, MG: UFV, 2005. .

MOURA, A.C.M. Reflexões metodológicas como subsídio para estudos ambientais baseados em análise multicritérios. In: SIMPÓSIO BRASILEIRO DE SENSORIAMENTO REMOTO, 13. (SBSR). 2007, Florianópolis. Anais... São José dos Campos: INPE, 2007, p.2899-2906.

NASCIMENTO, D. M. C.; DOMINGUEZ, J. M. L. Avaliação da vulnerabilidade ambiental como instrumento de gestão costeira nos municípios de Belmonte e Canavieiras, Bahia. Revista Brasileira de Geociências, v. 39, n. 3, p. 395-408, 2009.

NOAA. National oceanic and atmospheric administration.Vulnerability assessment: 1999. Disponível em: <www.csc.noaa.gov/products/nchaz/htm/tut. htm>. Acesso em: 24 jan. 2015.

ONU. Organização das Nações Unidas. Living with risk: A global review of disaster reduction initiatives. Inter-agency Secretariat International Strategy for Disaster Reduction (ISDR). Genebra: ONU, 2004.

RODRIGUEZ, J. M. M.; SILVA, E. V; CAVALCANTI, A. P. B. Geoecologia das paisagens: uma visão geossistêmica da análise ambiental. Fortaleza: UFC, 2004.

ROSS, J. L. S. Análise empírica da fragilidade dos ambientes naturais antropizados. Revista do Departamento de Geografia, São Paulo, v. 8, p. 63-74, nov. 1994.

SANTOS, R. D.; LEMOS, R. C.; SANTOS, H. G.; KER, J. C.; ANJOS, L. H. C. Manual de descrição e coleta de solo no campo. 5. ed. Viçosa: Sociedade Brasileira de Ciências do Solo, 2005..

SILVA, J. S. V.; SANTOS, R. F. Estratégia metodológica para zoneamento ambiental: a experiência aplicada na Bacia Hidrográfica do Alto Rio Taquari. Campinas: Embrapa Informática Agropecuária, 2011.

SPÖRL, C.; ROSS, J. L. S. Análise comparativa da fragilidade ambiental com aplicação de três modelos. GEOUSP: Espaço e Tempo (Online), São Paulo, n. 15, p. 39-49, junho 2004. TRICART, J. Ecodinâmica. Rio de Janeiro, RJ: IBGE/ SUPREN, 1977. .

VICENTE, L. E.; PEREZ FILHO, A. Abordagem sistêmica e geografia. Geografia, v. 28, n. 3, p. 323-344, 2003.

Saulo de Oliveira Folharini - Licenciado em Geografia pela Faculdade de Filosofia Ciências e Letras (FFCL), de São José do Rio Pardo (2008), bacharel em Geografia pela Universidade Estadual de Campinas (Unicamp), com mestrado em Geografia (2015) pela Unicamp. Atualmente é doutorando em Geografia pela Universidade Estadual de Campinas. 
Regina Célia de Oliveira - Bacharela e licenciada em Geografia pela Universidade Estadual Paulista Júlio de Mesquita Filho (1996), com mestrado em Engenharia Hidráulica e Saneamento pela Universidade de São Paulo (1999) e doutorado em Geociências e Meio Ambiente pela Universidade Estadual Paulista Júlio de Mesquita Filho (2003). Atualmente é professora da Universidade Estadual de Campinas.

André Luiz dos Santos Furtado - Licenciado e bacharel em Ciências Biológicas pela Universidade do Estado do Rio de Janeiro (1987, 1988), com mestrado em Ecologia pela Universidade Federal do Rio de Janeiro (1994) e doutorado em Ecologia pela Universidade Federal do Rio de Janeiro (2000). Atualmente é pesquisador da Empresa Brasileira de Pesquisa Agropecuária.

\section{Contribuições dos autores}

Os autores trabalharam conjuntamente com contribuições científicas e intelectuais ao estudo. O primeiro autor realizou o processamento, interpretação dos dados e preparação inicial do manuscrito. O segundo autor colaborou com a discussão dos resultados, oferecendo substanciais contribuições quanto à interpretação dos dados. O terceiro autor contribui na tabulação e interpretação dos dados e discussão acerca da fundamentação teórica.

Recebido para publicação em 07 de março e 2017

Aceito para publicação em 04 de maio de 2017 